\title{
Improving resolution in large telescopes
}

Vidal F. Canales, Pedro J. Valle, Manuel P. Cagigal, and Nicholas Devaney

Using simple filters at the telescope aperture overcomes diffraction limits on the resolution of astronomical images.

New discoveries in astronomy require the use of ever larger telescopes. There are two motives for increasing the telescope aperture: greater light-gathering power and the potential for higher spatial resolution. The largest of these telescopes require segmented mirrors, since current technology does not provide for the fabrication of monolithic mirrors with diameters much larger than $8 \mathrm{~m}$. In addition, random errors in the positioning of the segments will give rise to random speckle effects in the astronomical images, which complicates the detection of faint structures. It would therefore be desirable to eliminate these adverse effects while maintaining the advantages of having a large aperture. ${ }^{1}$

A possible solution for the static artifacts is to place a mask at the pupil plane of the telescope. There are many different proposals in the literature for masking. Some of them place a mask in each individual segment of the aperture. ${ }^{2}$ Others are based on masking the full telescope aperture. Some approaches propose transmittance masks, whereas others prefer phase elements. In this work, we use circularly symmetric supergaussian amplitude masks in the full segmented aperture in order to improve image quality.

We chose the supergaussian profile for two reasons: it avoids sharp edges, thereby reducing diffraction effects, and it provides some degree of control over the shape of the point spread function (PSF), as shown in Figure 1. These supergaussian filters can be implemented dynamically using a spatial light modulator, or can be fabricated, for example, in High Energy Beam Sensitive (HEBS) glass (Canyon Materials), which reacts to electron-beam exposure. Varying the exposure allows the desired supergaussian profile to be written into the glass with very high resolution in position and intensity.

Unfortunately, telescopes never yield an ideal performance, owing to random piston and tip-tilt errors that affect the placement of each segment, gaps between the segments, distorted segment edges, and so on. Nonetheless, supergaussian filters can im-
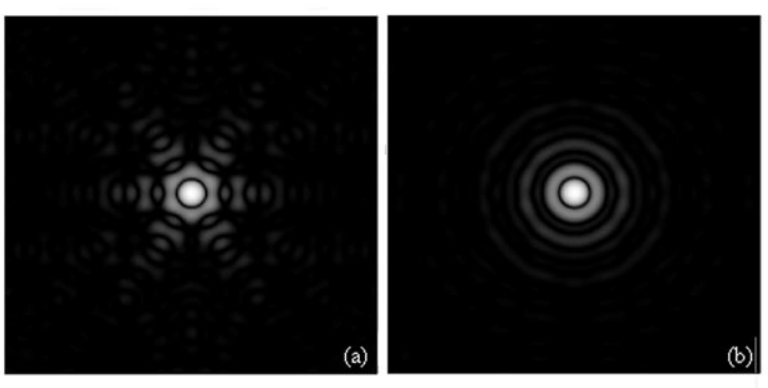

Figure 1. A supergaussian amplitude mask can control the shape of the point spread function (PSF). On the right is the PSF of a segmented telescope formed by two rings of hexagonal segments (of size a) and a central obscuration (a). The PSF can become circularly symmetric by placing at the pupil an amplitude supergaussian mask with standard deviation $\sigma=0.4 a$ and coefficient $\alpha=12(b)$. The log scale is used. The Strehl ratios are $1(a)$ and 0.69 (b).

prove resolution and eliminate the static diffraction pattern due to the segmented telescope geometry even in the presence of moderate error sources. We have compared annular and supergaussian masks with different error sources: piston errors, tip-tilt errors, both piston and tip-tilt errors, and gaps between segments. We have checked that the supergaussian filter with exponent $\alpha=12$ presents low sidelobes and the best Strehl ratio in every case, although different supergaussian filters may be useful in other circumstances. As an example, Figure 2 shows the comparison of several filters for the case of both piston and tip-tilt errors.

Furthermore, the majority of large telescopes are equipped with adaptive optics (AO) systems that adjust the mirrors to compensate for atmospheric distortions. However, it is impossible to obtain perfect adaptive compensation due to several error sources, including fitting errors and temporal delay between sensing and compensation. As a consequence, the compensated PSF consists of a coherent core surrounded by a speckled halo. The height of this residual halo depends on the turbulence strength and the AO system characteristics. It is therefore

Continued on next page 


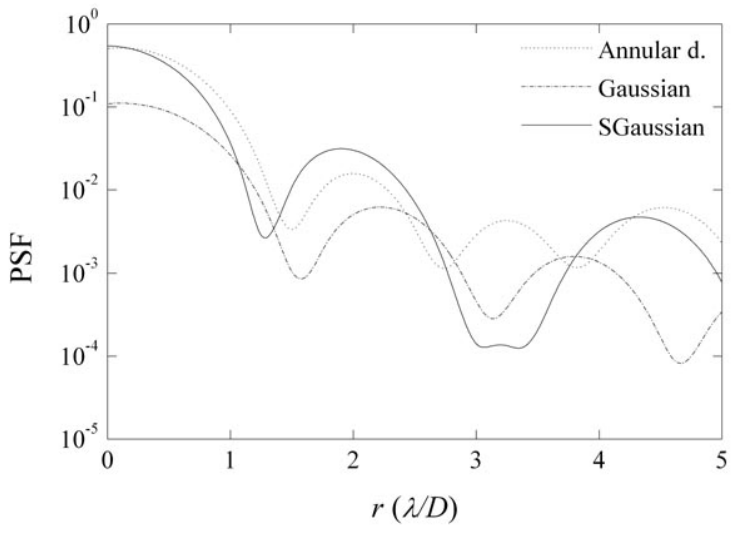

Figure 2. This comparison shows the radial profile of the PSF obtained with piston and tip-tilt errors using an annular diaphragm (dotted curve) and two supergaussian masks, $\sigma=0.2 a$ with $\alpha=1$ (dotteddashed curve), and $\sigma=0.4 a$ with $\alpha=12$ (solid curve).

sufficient to reduce the diffraction pattern using supergaussian filters to a level below the halo that remains after partial correction by the AO system.

In summary, we have analyzed the use of supergaussian amplitude filters to decrease the structure pattern due to the segmentation geometry of large telescope mirrors. In addition, we have discussed how the characteristics of the AO system must be considered when choosing the filter. The future development of this technique faces several challenges. First, a thorough study of the instrument characteristics and limitations should be carried out (e.g., polishing and phasing errors, and random gaps between segments). Second, supergaussian filters need to be combined with complex filters that yield superresolution to attain the resolution predicted for larger telescopes. ${ }^{3}$

This research was supported by the Ministerio de Ciencia y Tecnología grant AYA2004-07773-CO2-01

\section{Author Information}

\section{Vidal F. Canales}

Departamento de Física Aplicada

Universidad de Cantabria

Santander, Spain

http:/ / www.optica.unican.es

Vidal F. Canales is an assistant professor of physics at the University of Cantabria, Spain, who received his $\mathrm{PhD}$ degree from the same university in 2000. He has worked in target recognition and adaptive optics systems analysis in astronomical telescopes.
His current research interests include novel wavefront sensing techniques and filter design.

Pedro J. Valle and Manuel P. Cagigal

Departamento de Física Aplicada

Universidad de Cantabria

Santander, Spain

Pedro J. Valle received MS and PhD degrees in applied physics at the University of Cantabria in 1989 and 1991, respectively. He has worked on tunable birefringent filters, light scattering by composite surfaces, and surface polaritons. Since 2000, he has been working in PSF engineering.

Manuel P. Cagigal received his PhD in 1982 from the University of Cantabria. In 1982 he joined the university, where he is now a professor of optics. His research work was initially the analysis of light scattered from macromolecule solutions. His current research interests deal with adaptive optics. He is a member of SPIE and OSA.

\section{Nicholas Devaney}

Department of Experimental Physics

National University of Ireland

Galway, Ireland

Nicholas Devaney is a lecturer in the Department of Experimental Physics at the National University of Ireland, Galway. He has worked at the Royal Greenwich Observatory (19891991), Meudon Observatory (1991), and the Instituto de Astrofísica de Canarias (1992-2005). Current research interests include techniques for exoplanet detection and multiconjugate adaptive optics.

\section{References}

1. M. Troy and G. Chanan, Diffraction effects from giant segmented-mirror telescopes, Appl. Opt. 42, pp. 3745-3753, 2003.

2. R. Angel, J. Burge, J. Codona, W. Davison, and B. Martin, 20 and 30m telescope designs with potential for subsequent incorporation into a track-mounted pair (20/20 or 30/30), Proc. SPIE 4840, pp. 183-193, 2003.

3. V. F. Canales, D. M. de Juana, and M. P. Cagigal, Superresolution in compensated telescopes, Opt. Lett. 29, pp. 935-937, 2004. 